\title{
Pascal Brioist, Les Audaces de Léonard de Vinci
}

Paris, Stock, 2019

\section{Liliane Hilaire-Pérez}

\section{CpenEdition} Journals

Édition électronique

URL : http://journals.openedition.org/artefact/6137

DOI : $10.4000 /$ artefact.6137

ISSN : 2606-9245

Éditeur :

Association Artefact. Techniques histoire et sciences humaines, Presses universitaires du Midi

\section{Édition imprimée}

Date de publication : 15 juillet 2020

Pagination : 359-365

ISBN : 978-2-8107-0691-4

ISSN : 2273-0753

\section{Référence électronique}

Liliane Hilaire-Pérez, "Pascal Brioist, Les Audaces de Léonard de Vinci », Artefact [En ligne], 12 | 2020, mis en ligne le 21 décembre 2020, consulté le 23 décembre 2020. URL : http://

journals.openedition.org/artefact/6137 ; DOI : https://doi.org/10.4000/artefact.6137

Ce document a été généré automatiquement le 23 décembre 2020.

\section{(9) $\odot \Theta$}

Artefact, Techniques, histoire et sciences humaines est mise à disposition selon les termes de la Licence Creative Commons Attribution - Pas d'Utilisation Commerciale - Pas de Modification 4.0 International. 


\section{Pascal Brioist, Les Audaces de Léonard de Vinci}

Paris, Stock, 2019

Liliane Hilaire-Pérez

\section{RÉFÉRENCE}

Pascal Brioist, Les Audaces de Léonard de Vinci, Paris, Stock, 2019, 473 p.

1 Pascal Brioist, professeur d'histoire moderne à l'université de Tours, est un spécialiste international des études léonardiennes. Il doit sa notoriété à des recherches novatrices qui ont mis en valeur la place centrale des techniques militaires dans l'œuvre de Léonard ${ }^{1}$, mais aussi aux multiples manifestations scientifiques qu'il a lancées. Depuis une quinzaine d'années, Pascal Brioist est à l'origine de colloques, d'expositions et de films documentaires révélateurs d'une ambition pédagogique, d'une créativité et d'une capacité à fédérer des collaborations pour diffuser une image renouvelée de Léonard de Vinci à la lumière des perspectives les plus récentes en histoire des sciences et en histoire des techniques ${ }^{2}$. C'est cette remarquable articulation entre niveaux d'analyse et de compréhension d'une part, et renouvellements historiographiques de l'autre qui fait l'originalité de ce livre.

2 D'un côté se tient la question du génie, posée dès l'introduction. Elle traverse le livre et sous-tend la conclusion qui se clôt par une réflexion sur " la pensée héroïque ", en écho à l'intitulé de la collection de l'éditeur Stock. L'idée de génie est prise au sérieux par Pascal Brioist qui, s'il rappelle ses mises en cause, de Marcellin Berthelot à Bertrand Gille et à Paolo Galluzzi - dont l'excellent catalogue d'exposition exprimait avec brio la pertinence des concepts de communauté d'ingénieurs et d'invention collective ${ }^{3}$ analyse la construction du mythe léonardien tout en interrogeant la manière de rendre compte des qualités d'exception d'un individu. Loin d'héroïser Léonard, Pascal Brioist décrit la complexité d'une pensée ramifiée et synthétique portée par une culture hybride, pratique, lettrée et mathématique. En ce sens, Pascal Brioist inscrit sa 
réflexion dans le courant d'étude qui a placé la mixité des savoirs au cœur du renouvellement de l'histoire des techniques et de l'histoire des sciences, particulièrement pour la période moderne, de Bernard de Palissy chez Pamela H. Smith aux artisanal-scientific experts d'Ursula Klein ${ }^{4}$. On est encore loin d'avoir perçu l'ampleur des résonances entre les hybridations culturelles, que l'on peut suivre à travers la formation, les contacts, les voyages, les bibliothèques, et l'élaboration de raisonnements et de visions du monde, en quelque sorte de modes de connaissance, d'épistémê dont les traces sont à chercher aussi bien dans les traités que dans les notes, les dessins, les réalisations et les œuvres de l'esprit, des matériaux qu'il convient d'analyser comme une " histoire concrète de l'abstraction " selon la formule de JeanClaude Perrot. Bien des travaux sont mobilisés et c'est un tour de force de réunir en une synthèse accessible des recherches complexes et foisonnantes sur les savoirs à la Renaissance, depuis les auteurs phares comme Daniel Arasse, Carlo Pedretti, Romano Nanni, jusqu'aux jeunes chercheure's dans des domaines les plus variés, Andrea Bernardoni et Alexander Neuwhal sur les machines de chantier, Marjolijn Bol sur les huiles et les vernis, Viktoria Tkaczyk sur les machines de théâtres etc. Proposer une relecture de l'œuvre de Léonard impose éclectisme et érudition puisqu'il s'agit de comprendre la polyvalence des compétences, les transferts de savoirs, les analogies et les correspondances, en somme une pensée substitutive et synthétique qui marque durablement la culture technique en Europe et traverse, voire recompose les mondes sociaux autour de grammaires de l'agir.

On comprend que la question du génie, au-delà d'une approche en termes de contextualisation, faisant la part des collectifs et des transmissions, y compris dans l'atelier, au-delà aussi d'une histoire du façonnage de soi que Pascal Brioist mobilise excellemment à plusieurs reprises pour éclairer la distanciation de l'ingénieur-artiste avec le monde des métiers, ouvre sur l'étude d'une pensée, "la pensée héroïque ", c'est-à-dire pour l'auteur, "celle du sage », capable de peser les avantages et les contraintes, de composer, de réduire l'adversité, signe comme le disait Hélène Vérin que "L'espace de la délibération préalable à la réalisation s'ouvre indéniablement ${ }^{5}$ ». Pour Pascal Brioist, c'est «un mode de pensée profond où l'hésitation précède nécessairement le choix du trait juste» (p.472). Ce constat qui conclut le livre est fondamental. Il permet de mettre en perspective les interprétations de l'œuvre de Léonard, les unes contribuant au mythe du génie, les autres critiques à l'égard d'un esprit fertile mais "velléitaire ", «incapable de réaliser ses intentions" - un lieu commun aussi dont ont souffert les théâtres de machines longtemps vus comme des élucubrations ${ }^{6}$. C'est en qualifiant la pensée de Léonard et son intellection du monde que Pascal Brioist réfute les interprétations hâtives et les poncifs éculés. La démonstration est pleinement convaincante et montre que les apports récents de l'historiographie doivent inciter à rouvrir des sujets, même plus canoniques. C'est l'audace de Pascal Brioist.

D'un autre côté, il ne s'agit en aucun cas d'un ouvrage savant, du moins pas uniquement.

5 La performance que constitue ce livre se situe aussi dans le défi biographique qui restitue en un récit commun, partageable, compréhensible le parcours intellectuel d'un créateur hors norme. Loin d'une biographie intellectuelle, c'est bien l'individu dans l'infinie complexité que livrent les indices les plus infimes que suit Pascal Brioist. Le fil rouge du livre, ce sont «les énigmes" de Léonard, comme le souligne l'introduction, 
une manière de mettre en récit un parcours au ras de l'individu dans la société toscane du Xve siècle.

6 Le premier chapitre, «Souvenirs d'enfance " plonge dans l'histoire contrariée d'un fils illégitime mais entouré, une enfance ponctuée par des apprentissages pratiques, au contact du monde rural puis à Florence où Léonard découvre aussi bien les mathématiques que les décors imités de l'Antique. Il appartient dès ses débuts à « cette strate culturelle intermédiaire entre les lettrés et les non-lettrés", comme Filippo Brunelleschi (p. 61). Le chapitre suivant, «La Florence des ateliers " guide le lecteur au cœur de cet univers savant et populaire, à commencer par l'atelier du peintre Andrea Del Verrochio où Léonard entame une nouvelle formation. L'originalité, ce sont les transferts de savoir-faire, l'acquisition des techniques du dessin - l'anatomie, la perspective - en même temps que la connaissance des supports et des enduits. Les réalisations sont multiples, entre effets spéciaux, machines de spectacles, peintures saintes, portraits mondains, jusqu'au travail dans le «jardin-laboratoire » de Laurent de Médicis (p. 91). Pascal Brioist met en valeur la richesse de ces années de formation qui laissent cependant Léonard aux portes du savoir lettré et le placent aussi aux marges de la société en raison de son homosexualité. Dans le troisième chapitre, «Des lectures à l'écriture: un autodidacte d'exception", l'auteur approfondit la mixité culturelle qui caractérise Léonard et qui donne la clé de son œuvre. Il analyse les lectures de Léonard et le gonflement de sa bibliothèque, jusqu'à 123 volumes pendant son séjour à Milan, en même temps que les ouvrages évoluent des grammaires aux livres de sciences, avec apprentissage du latin, signes d'un façonnage de soi comme d'une inscription dans un milieu savant de professeurs, de médecins, de franciscains, d'ingénieurs (p.111). La rencontre décisive avec le mathématicien Luca Pacioli, franciscain, a lieu à la cour de Ludovic Le More en 1496.

Cette "hybridité de la culture de l'artiste-chercheur» (p.120) permet de comprendre la place de Léonard dans "la tradition des arts mécaniques", analysée dans le quatrième chapitre. Rappelant le rôle de l'atelier de Verrochio dans la confection de la lanterne du Dôme de Florence, Pascal Brioist élargit le panorama jusqu'à voir dans la Toscane, un "laboratoire de mécanique ", montrant que Léonard se forme grâce à un fonds commun de savoirs, ceux de "l'ingénierie toscane" que promeut un milieu d'ingénieurs via des formes multiples de communication, par l'écrit et le dessin, dans l'atelier, par les voyages. Cette profusion de savoirs, appliqués à des réalisations les plus variées ouvre la voie à une rationalisation, indissociable du « processus de réduction en art» qui marque la culture technique de la Renaissance. Pour rendre son propos percutant, Pascal Brioist rappelle les études récentes qui proposent une filiation entre les typologies mécaniques de Léonard et celles de Franz Reuleaux au début du $\mathrm{xx}^{\mathrm{e}}$ siècle. Le cinquième chapitre, "L'observation, la pensée analogique et les proportions ", analyse les prolongements de cette pensée comparative et synthétique, qui nourrit une abstraction née de l'observation du réel. À partir des analogies de formes et de fonctions entre l'homme, l'animal et les phénomènes naturels, Léonard pense le monde comme système, cherchant des lois naturelles dont l'identification est une des clés de la mise en cause des hiérarchies aristotéliciennes. Mais plus que des positions philosophiques, c'est le rapport à l'expérience et à l'observation qui conduit Léonard à prendre de la distance avec les héritages conceptuels et à « faire bouger les lignes épistémologiques»(p.216). Cette innovation concerne le sixième chapitre "Léonard, de l'expérimentation à la théorisation». Pascal Brioist rappelle avec 
beaucoup de clarté la polysémie du terme « expérience » à la Renaissance, tendu entre la vérification de théories, le vécu et l'intuition (experientia), les raisonnements inductifs ouvrant sur les protocoles de démonstration (experimentum). Si ce dernier sens, novateur, existe chez Léonard, la valeur d'une expérience tient toujours pour lui dans « la mise à l'épreuve d'une théorie abstraite » (p. 227), signe d'un "savoir mixte ", réfutant « la science purement mentale » et « la pratique sans théorie» (p. 234). Cette capacité d'articuler l'observation et la théorisation sous-tend les essais de modélisation des phénomènes physiques et la remise en cause des auteurs avérés, sur les couleurs, la physiologie, la géologie. Elle lui permet de prendre place aussi dans les débats sur les principes du mouvement qui traversent les mathématiques à la Renaissance, notamment dans leurs usages militaires ${ }^{7}$.

Les chapitres suivants reprennent le fil des pérégrinations de Léonard et de son ascension sociale, non sans écho aux livres célèbres de Mario Biagioli et Matteo Valleriani sur Galilée ${ }^{8}$, courtisan et ingénieur. Le septième chapitre, "Comment devient-on courtisan quand on n'est pas bien né » est consacré à l'entrée de Léonard au service des Sforza à Milan après son départ de Florence en 1482. Ludovic Le More offre à Léonard des conditions idéales pour développer son atelier dans l'ancien château des Visconti. Les réalisations sont multiples, peintures, maquettes ainsi pour la tourlanterne du Dôme de Milan, fêtes et machines de spectacles, jusqu'à la chute des Sforza. Alors commence le lien avec les rois de France, Charles VIII, puis Louis XII qui envahit le Milanais en 1499. Les guerres d'Italie sont une étape cruciale dans la carrière de Léonard, devenu "peintre et ingénieur ordinaire du Roi de France " sous Louis XII (p. 320). Le ralliement à Louis XII, véritable trahison, lui donne l'occasion, en 1502, d'entrer au service de César Borgia, neveu du pape Alexandre VI, duc du Valentinois et nouveau chef des armées florentines. C'est Florence (et Machiavel) qu'il sert à nouveau après la chute de Borgia, puis le gouverneur de Milan, Charles d'Amboise (1507). Le duché de Milan est cependant perdu par la France en 1512 et Léonard commence un séjour à Rome, dont le nouveau pape en 1513 est un Médicis, jusqu'à ce que la bataille de Marignan modifie les équilibres et lui donne l'occasion de rencontrer François $\mathrm{I}^{\mathrm{er}}$ à Bologne, à la fin 1515. Ces itinéraires curiaux se doublent d'une carrière d'ingénieur militaire. C'est le thème du huitième chapitre, "Comment devient-on ingénieur militaire?». Pendant la période milanaise, Léonard s'aguerrit auprès d'experts militaires, notamment pour les fortifications et bénéficie des atouts de Milan, capitale de l'armement (armurerie, fonderie, artillerie). Les armes deviennent son champ privilégié de recherche et ses réalisations «laissent leur empreinte » (p. 357). César Borgia le fait " architecte et ingénieur général », chargé de l'inspection des forteresses des Marches, une occasion de lancer des chantiers et de construire des engins. Les menées guerrières de Borgia lui offrent des opportunités pour produire des armes et des machines de siège dans ses ateliers des faubourgs de Rome. Au service de Florence, c'est la dérivation de l'Arno lors de la guerre contre Pise (1504) et la fortification de Piombino. La vie de Léonard est " traversée par les guerres d'Italie », comme l'exprime son œuvre disparue, La Bataille d'Anghiari. C'est aussi un traumatisme selon Pascal Brioist. Le neuvième chapitre, «Faire de l'art du peintre une science et de l'artiste un démiurge" renoue avec les interrogations sur les ressorts de la création et la construction du génie. Pour Léonard, la peinture est un art libéral, indissociable des mathématiques; elle est aussi un art de la composition, nourri de variations dans un répertoire de motifs, y compris ceux qu'offre la nature. Cette pensée synthétique, à l'œuvre dans les résonances du paysage et du visage de La Joconde, fait du peintre un 
penseur, un inventeur et un expérimentateur (p. 417). Loin du génie isolé, Léonard "se livre à une apologie en règle du travail d'atelier » (p.418), il fait école et s'entoure de deux disciples lors de son départ pour la France. Le dernier chapitre, intitulé « L'ami du roi », analyse les conditions du séjour de Léonard au Clos Lucé. Le contexte du départ, ainsi la concurrence de Michel-Ange, la mort du Julien de Médicis, le projet de Louise de Savoie d'un palais à Romorantin est restitué en détail, et Pascal Brioist évoque jusqu'au voyage de Léonard convoyant livres, folios, peintures, pièces mécaniques. L'installation, les émoluments, l'estime du roi, la visite de Louis d'Aragon, les fêtes et les spectacles militaires, le chantier inachevé de Romorantin: dans ce chapitre, on perçoit la passion de l'auteur qui a fait revivre avec tant d'intensité les dernières années de Léonard à Amboise pour le plus grand plaisir des étudiants de Tours, qu'il remercie, des chercheurs et du public.

Présenter la vie de Léonard comme un objet d'étude renouvelé, susceptible d'interprétations multiples et de relectures à la lueur des derniers acquis de l'histoire était une gageure. Placer les sciences et les techniques au cœur de la réflexion, et comprendre par cette voie, Léonard comme un hybride culturel en était une autre. Enfin, partir du questionnement du génie, un thème commun, et en faire la source d'une réflexion sur la " pensée héroïque » comme pensée de la délibération, de l'essai et de l'expérimentation représente le défi le plus fort de ce livre magistral.

\section{BIBLIOGRAPHIE}

BIAGIOLI Mario, Galileo, Courtier: The Practice of Science in the Culture of Absolutism, University of Chicago Press, Chicago, 1993.

BRIOIST Pascal, Léonard de Vinci, homme de guerre, Alma éditeur, Paris, 2013.

DOLZA Luisa et VÉRIN Hélène, « Figurer la mécanique : l'énigme des théâtres de machines de la Renaissance ", Revue d'histoire moderne et contemporaine, 51-2, avril-juin 2004, p. 7-37.

GALLUZZI Paolo, Les Ingénieurs de la Renaissance de Brunelleschi à Léonard de Vinci, Cité des Sciences et de l'Industrie/Finmeccanica, Paris/Florence, 1995.

KLEIN Ursula, « Artisanal-scientific Experts in Eighteenth-century France and Germany », Annals of Science, 2012, 69/3, p. 303-306.

SMITH Pamela H., The Body of the Artisan. Art and Experience in the Scientific Revolution, University of Chicago Press, Chicago, 2004.

VALLERIANI Matteo, Galileo Engineer, Springer, Dordrecht, 2010.

VÉRIN Hélène, La Gloire des ingénieurs. L'intelligence technique du XVI ${ }^{e}$ au XVIII siècle, Albin Michel, Paris, 1993. 


\section{NOTES}

1. Brioist, 2013.

2. Exposition "Les rêves mécaniques de Léonard de Vinci... des croquis aux machines ", Espace culturel de Rombas en Moselle (20 janvier-20 avril 2008) ; colloque international « Léonard de Vinci, artiste et ingénieur de François $\mathrm{I}^{\mathrm{er}}$ », Romorantin (3-5 juin 2010) et exposition « Léonard de Vinci : Romorantin, le projet oublié » (9 juin 2010-30 janvier 2011); exposition " Une idée mille machines de Léonard de Vinci à Jean Errard", Musée de l'histoire du fer, Jarville (4 mai 2013-5 janvier 2014) ; "Marignan 1515/2015» (juillet 2015), reconstitution d'une fête de Cour de 1518 célébrant la victoire de Marignan et le roi François $\mathrm{I}^{\mathrm{er}}$ (parc Léonard de Vinci du Clos Lucé, Amboise et parc de Beauvais, Romorantin) https://www.marignan2015.fr/; webdocumentaire interactif «Sur les pas de Léonard» https://renaissance-transmedia-lab.fr/webdocumentaire/ ; $62^{\mathrm{e}}$ colloque international d'études humanistes, «Léonard de Vinci : invention et innovation» (Tours, Amboise, Chambord, 24 juin-28 juin 2019); exposition «S'inspirer du vivant : le biomimétisme de Léonard de Vinci à nos jours » (Fabrique Normant, $1^{\mathrm{er}}$ juillet-31 août 2019), dans le cadre de l'année 2019 «Viva Leonardo Da Vinci - 500 ans de Renaissance(S) en Centre-Val de Loire ».

3. Galluzzi, 1995.

4. Smith, 2004 ; Klein, 2012.

5. Vérin, 1993, p. 27.

6. Dolza, Vérin, 2004.

7. Brioist, 2013.

8. Biagioli, 1993 ; Valleriani, 2010.

\section{AUTEURS}

\section{LILIANE HILAIRE-PÉREZ}

Université de Paris (ICT) / EHESS (CAK) / IUF 